\title{
The small airways in asthma
}

\author{
Anita Trikha $\cdot$ Richard J. Martin
}

Published online: 5 October 2013

(C) Springer Science+Business Media New York 2013

\begin{abstract}
Airway inflammation is the basis for airway hyperreactivity that results in the signs and symptoms of asthma. Great strides have been made in the past several decades to target airway inflammation using topical inhaled corticosteroids. In some cases, despite reported improvement in large airways indices on treatment, clinical control of asthma is not attained. Inflammation of the distal airways is now realized as playing a greater role in asthma control, and newer techniques are available to assess the smaller airways. Changes in formulations of inhaled corticosteroids and smaller particle size of medication allows for more effective targeting of the smaller airways.
\end{abstract}

Keywords Small airways $\cdot$ Inhaled corticosteroid particle size $\cdot$ Asthma

\section{Introduction}

Asthma is common disorder that is clearly now seen as heterogeneous, both in presentation and response to treatment. Traditionally, treatment has been based on measurements of large and medium-sized airways (those $2-4 \mathrm{~mm}$ in diameter). More recently, insight into the involvement of the distal airways in asthma has brought to light the possibility of the small airways (as defined as those distal to the seventh or eighth generation of the bronchial tree with an inner diameter of $<2 \mathrm{~mm}$ [1]) playing a

A. Trikha $\cdot$ R. J. Martin $(\bowtie)$

The Department of Medicine, National Jewish Health,

1400 Jackson Street, Denver, CO 80210, USA

e-mail: MartinR@NJHealth.org

A. Trikha

e-mail: TrikhaA@NJHealth.org

A. Trikha

Division of Allergy and Immunology, National Jewish Health,

Denver, CO, USA role in the variability of presentation and as a target for treatment. However, to prove that distal (small) airway dysfunction and inflammation is involved in a given asthma patient may be difficult, as it involves a complex set of studies. Thus, it is uncommon for caregivers to consider the distal airways in treatment decisions. This may lead to less control for the patient. One of the possibilities to help interpret why recent nationwide surveys show that overall our asthma population is not well controlled is the lack of attention to what is occurring in the distal lung. The Real World Evaluation of Asthma Control and Treatment Study $(n=1,788)$ [2] used the Asthma Control Test to demonstrate that $55 \%$ of the population is poorly controlled or not well controlled. In 1998, the Asthma in America study [3] documented in a nationwide survey that asthma patients had $10 \%$ hospitalization rates, $20 \%$ limitation on activity, $22 \%$ limitation with work, $26 \%$ missed work/school, and $37 \%$ acute care visits. A decade later, with emphasis on the use of inhaled corticosteroids, the Asthma Insight and Management study ( $n=2,294)$ [4] demonstrated some improvement over the Asthma in America study, but nothing dramatic. Furthermore, a well-designed 1-year study, Gaining Optimal Asthma controL (GOAL), using up to maximum inhaled steroid dosing in 3,421 patients, demonstrated that approximately $75 \%$ of all patients achieved the designation of well-controlled [5]. Thus, $25 \%$ still were not well-controlled. Although adherence/compliance as well as other issues certainly can play a marked role in the ability to control asthma, the distal airways must also be considered in every asthma patient.

\section{Anatomy}

The surface area of the airways is equal to approximately half of a tennis court, with the vast majority of this area represented by the small (distal, peripheral) airways. Most of the 
inflammatory and physiologic changes in asthma occur in the distal airways, as noted in the rest of this review article.

In asthmatic individuals, the anatomic outer wall area has been demonstrated to be thicker in the smaller airways $(<2 \mathrm{~mm})$ than in larger airways (those $2-4 \mathrm{~mm}$ in size) [6]. In this postmortem study, both non-fatal (died from causes other than asthma), and fatal asthma had outer wall thickness in the small airways that was significantly thicker compared to controls. Of interest, there was no difference in airway thickness (outer wall or total wall area) between the asthma groups when looking at the $2-4 \mathrm{~mm}$ airways [6].

\section{Inflammation}

Nocturnal asthma (NA) has been a good model for investigating the small airways. Hetzel and colleagues have demonstrated that the nadir of lung function in NA is 4:00 AM, and that the majority of deaths and respiratory arrests due to asthma occur during the night $[7,8]$. Transbronchial biopsies (distal airways) taken from patients with nocturnal asthma have demonstrated that there is significant inflammation in the alveolar area, mainly consisting of eosinophils in the infiltrate, although other inflammatory cells demonstrate the same circadian trend [9]. This finding was most significant in the biopsies obtained at 4:00 AM (Fig. 1) compared to 4:00 PM. The alveolar tissue inflammation, but not the proximal larger airway inflammation, correlated inversely with the fall in lung function. T cells have the ability to recruit eosinophils via the production of cytokines such as IL-4, IL-5 and IL-13, as well as chemokines such as eotaxin and RANTES, and there is a presence of CD4+ cells in alveolar tissue small airways biopsy [9]. There is also a similar relationship with lung function, i.e., increased number of CD4+ cells and decreased lung function [9].

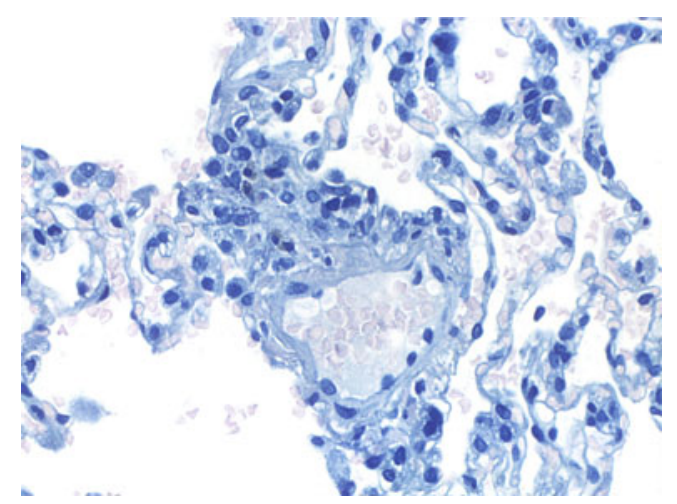

Fig. 1 Distal airway inflammation in nocturnal asthma. This is a transbronchial biopsy from a nocturnal asthmatic taken at 4:00 AM demonstrating the distal airway. The open spaces are the alveolar areas. Note the tremendous inflammatory infiltration in the areas around the alveolar spaces. The cells represent all inflammatory types of cells and are greatly increased from 4:00 PM (not shown)
There have been studies looking at the differences in inflammation between the larger ( $>3 \mathrm{~mm}$ outer perimeter) and smaller airways. Autopsy studies of asthmatics have shown that the location of the inflammatory cells is different in the larger airways as compared to the smaller airways. In the larger airways in asthma, inflammatory cells are found inbetween the smooth muscle and the sub-basement membrane, referred to as inner leukocyte density staining. In comparison, outer leukocyte density staining is found in the smaller airways, with inflammatory cells found between the smooth muscle and the alveolar attachments [10]. This has the potential to affect airway constriction by different mechanisms: sub-mucosal inflammation being associated with increased luminal occlusion with muscle shortening versus adventitial inflammation promoting airway constriction by decreased tethering of the airways [11]. Haley and colleagues also demonstrated that cystic fibrosis patients had a different pattern of distribution of inflammatory cells in their smaller airways, perhaps signifying a distinction between asthma and other obstructive diseases (in cystic fibrosis, eosinophils were fewer in number and were more often present in the larger airways) [10]. These differences in patterns of inflammation could be a result of differential recruitment related to etiology and disease-specific, regional organization of pulmonary inflammation [10]. For example, allergen challenge studies done in vitro have also demonstrated a greater release of mediators such as histamine, prostaglandins and thromboxane B2 in lung parenchyma when compared to large airways [12]. This again supports the theory that local release of cytokines and chemoattractants influences localization of inflammation. In fact, Hauber and colleagues demonstrated that the eosinophilic infiltration in both the central and peripheral airways, as well as the elevation in IL-5mRNA and eotaxin mRNA seen in asthmatics, was suppressed in patients being treated with hydrofluoroalkane (HFA)-flunisolide (an extra-fine particle steroid) and this was accompanied by increases in forced expiratory volume in the first second $\left(\mathrm{FEV}_{1}\right)$ and forced expiratory flow between $25 \%$ and $75 \%$ of expiration $\left(\mathrm{FEF}_{25-75}\right)$ [13].

\section{Physiology and evaluation techniques}

Studies done in the late 1960s birthed the term the "quiet zone" to describe the distal airways of the lung by demonstrating that the small airways $(<2 \mathrm{~mm})$ accounted for $<10 \%$ of total airflow resistance, due to the far larger surface area in the distal lung. The smaller airways lack the cartilaginous support system of the larger airways, which influences their response to inflammation. While increasing lung volume generally equates to increase in airway diameter producing a decrease in airway resistance of the larger airways, this does not hold true for the smaller airways [14]. This is possibly why the contribution of the small airways to disease control had not been appreciated earlier. 
Again using nocturnal asthma as a study model, studies have been performed evaluating the volume-resistance relationship, and these demonstrated an uncoupling of the volume-resistance relationship in the smaller airways in patients with NA during sleep [15]. In the normal lung, an increase in lung volume results in decreased airway resistance as lung parenchyma essentially stretches out the attached airways (airways coupled to the lung parenchyma). In asthma, because of inflammation and edema surrounding especially the smaller airways, there is a loss of this coupling between the airways and lung parenchyma, and thus the resistance remains unchanged despite increases in lung volumes.

Physiologically, peripheral airway resistance (PAR) can be measured using a bronchoscope wedged into a fifth or sixth generation airway. By changing air flow via one port and measuring change in pressure via another port in the bronchoscope, PAR can then be calculated. Studies done in nocturnal asthma subjects have shown that there is an increase in PAR, decrease in compliance and an increase in 4:00 AM plateau pressure (as compared to 4:00 PM) that suggests a loss or closure of collateral channels in the distal airways at that time [16].

Wagner and colleagues used this PAR technique to compare normal subjects to mild asthmatic subjects [17]. By increasing flow rates via the bronchoscope, they demonstrated that normal subjects did not have an increase in pressure, thus there was no increase in PAR ( $\mathrm{PAR}=\Delta$ pressure/ $\Delta$ flow). In subjects with mild asthma having normal or minimal impairment in spirometric values, the PAR was significantly increased. Thus, it is not just severe asthmatics that have the potential for distal airway dysfunction.

Spirometry is a simple, non-invasive method of assessing lung function. Measurements of forced vital capacity (FVC) and $\mathrm{FEV}_{1}$ are standardized based on age, height, sex and ethnicity. Reproducibility on testing is required to ensure reliability, as testing is fairly effort-dependent. In general, $\mathrm{FEV}_{1}$, FVC and the ratio are considered measures of larger airways. There are calculated measurements that are now considered to reflect small airway disease, such as closing volume (discussed below) and forced expiratory flow at $25-75 \%$ of FVC. Complete pulmonary function testing with measurement of residual volume (RV) can be an indicator of small airways disease. This is especially relevant in the setting of a normal $\mathrm{FEV}_{1} / \mathrm{FVC}$ ratio and reduced FVC [14]. Using a systemic medication, such as leukotriene inhibitors, correlates with a reduction in RV and improvement in symptoms [18]. Additionally, studies have demonstrated that asthmatics with more frequent exacerbations have higher RV at baseline than asthmatics with less frequent exacerbations [19].

In't Veen and colleagues studied children with asthma (with $\mathrm{FEV}_{1}$ of $93 \%$ ) categorized into two groups: those with year-round exacerbations and those without [20]. The only measurable difference between the two groups was the closing volume. The closing volume (CV) is defined as the volume of gas remaining in the vital capacity at the intersection of phase III and IV, and is usually expressed as percent of vital capacity (VC), CV/VC \%. The higher the CV, the earlier the closing of the distal airways. The elevation in the $\mathrm{CV}$ in the asthma exacerbation group suggests that these children have hyperreactive distal airways, and triggers such as viral infections, allergens, cold air, etc. produce closure of these units and induction of an exacerbation [20].

The forced expiratory flow 25-75, the M:P ratio (ratio of isovolumic maximal forced expiratory flow to partial forced expiratory flow at $60 \%$ of vital capacity), and PAR have all been suggested to assess distal lung function. In 1973, McFadden and colleagues demonstrated that despite symptom improvement after an acute exacerbation, there was continued small airway obstruction [21]. Interestingly, Zeidler et al. demonstrated that asthmatics exposed to cat allergen with a drop in their $\mathrm{FEV}_{1}$ to $20 \%$ below their baseline had persistent air trapping based on high-resolution computational tomography, FEF $25-75 \%$ and single breath nitrogen washout test 6 hours after allergen provocation, even though the $\mathrm{FEV}_{1}$ returned to baseline [22]. The FEF $25-75 \%$ is reproducible if lung volumes are the same, otherwise it is a variable measurement. These studies all support the idea that small airway changes play a role in the late-phase asthmatic response in patients with allergic asthma. Additionally, a lower FEF $25-75 \%$ has also been shown to correlate with an increase in exercise induced symptoms in asthmatics [23].

A study comparing these modalities and correlating them with evidence of inflammation on transbronchial biopsies was done by Sutherland et al. They demonstrated that in the severe asthmatics they studied, the degree of alveolar inflammation correlated poorly with the physiologic measures of FEF $25-75 \%$ and the M:P ratio. However, static lung volumes measured as thoracic gas volume and total lung capacity did correlate with distal lung eosinophilic infiltration [24].

Impedance oscillometry (IOS) to measure airways resistance was introduced in the 1950 s and became commercially available in in the 1990s. It is an effort-independent technique used to assess airway resistance, especially in children who are unable to perform spirometry in an acceptable manner. There are several components to the measurement of oscillometry. To simplify, low oscillation frequencies $(<15 \mathrm{~Hz})$ transmit more distally in the lung. Two airway resistance measures that are important in asthma are those taken at $20 \mathrm{~Hz}$ (R20), a reflection of large airway resistance as the sound waves do not travel distally, and at $5 \mathrm{~Hz}(\mathrm{R} 5)$, a measure of resistance of the overall respiratory system. Thus, R5 minus R20 (R5-20) is a measure of small airway resistance as it subtracts out the larger airway resistance from the total airway resistance. Additionally, the reactance area $(\mathrm{AX})$ is another measure of peripheral area function and helps define changes. AX is defined as the reactance (area under the curve) at all frequencies between $5 \mathrm{~Hz}$ and Fres (the frequency at which reactance is 0 ) [25•]. 
Using IOS to compare controlled to uncontrolled asthmatic children, one study assessed the utility of using several peripheral airway variables in asthma control and found that the R5-20 and AX were the best indicators of asthma control in these patients whereas the $\mathrm{FEV}_{1}$ did not correlate with asthma control [25•]. Small airways constriction is produced by histamine infusion and is reflected by changes of the R5-20 [26]. Thus, these studies have supported the utility of oscillometry as a technique to measure small airway resistance and reinforce the contribution of the small airways in asthma control. More severe exercise induced bronchospasm is also associated with a reduced R5-20 but not the R20, suggesting that exercise induced symptoms in asthmatics may be more closely related to changes in the smaller airways [27].

Fraction of exhaled nitric oxide ( $\mathrm{FeNO})$ is another newer indirect measure of inflammation in the respiratory tract. It is non-invasive, safe, simple and is mainly derived from the lower respiratory tract and produced by a reaction catalyzed by NO synthase. The inducible form of $\mathrm{N}$ synthase is produced in response to inflammatory mediators. FeNO is flow dependent and affected by many factors (e.g. smoking, ingestion of nitrate containing meats). There has been some difficulty in defining what a normal value is, even when flow rate is standardized at $50 \mathrm{ml} / \mathrm{s}$. FeNO is a surrogate measure used for monitoring airway inflammation and guiding steroid therapy in asthmatics [28•]. However, there have been mixed results in studies evaluating this as a marker for asthma control [29••]. A two-compartment model of NO exchange has been proposed, and an alveolar component of the NO can be calculated using regression analysis [30].

Alveolar nitric oxide is a reflection of distal lung inflammation. Berry et al. reported higher levels of alveolar NO in patients with refractory asthma as compared to normal subjects and those with mild-moderate asthma [31]. They demonstrated that alveolar NO responded to oral systemic steroids (which would target small airways) and not to doubling doses of large particle inhaled corticosteroids.

High resolution computed tomography (CT) of the chest allows examination of airways with a diameter as small as $1.5-2.0 \mathrm{~mm}$ and this has provided an ability to assess the small airways in asthma. Studies have demonstrated that airway thickening and hyper-reactivity occurs mainly in the small airways in patients with asthma [32].

Nitrogen washout testing may also be useful in assessing small airways inflammation, as it can distinguish between ventilation heterogeneity in the conductive versus acinar airways [33].

The clinical and measurable significance of the peripheral airway inflammation seen on pathological specimens has been difficult to assess. Table 1 summarizes parameters and techniques used to evaluate airway inflammation and function, as discussed above. There is no perfect or easy way to measure peripheral airway inflammation and function.

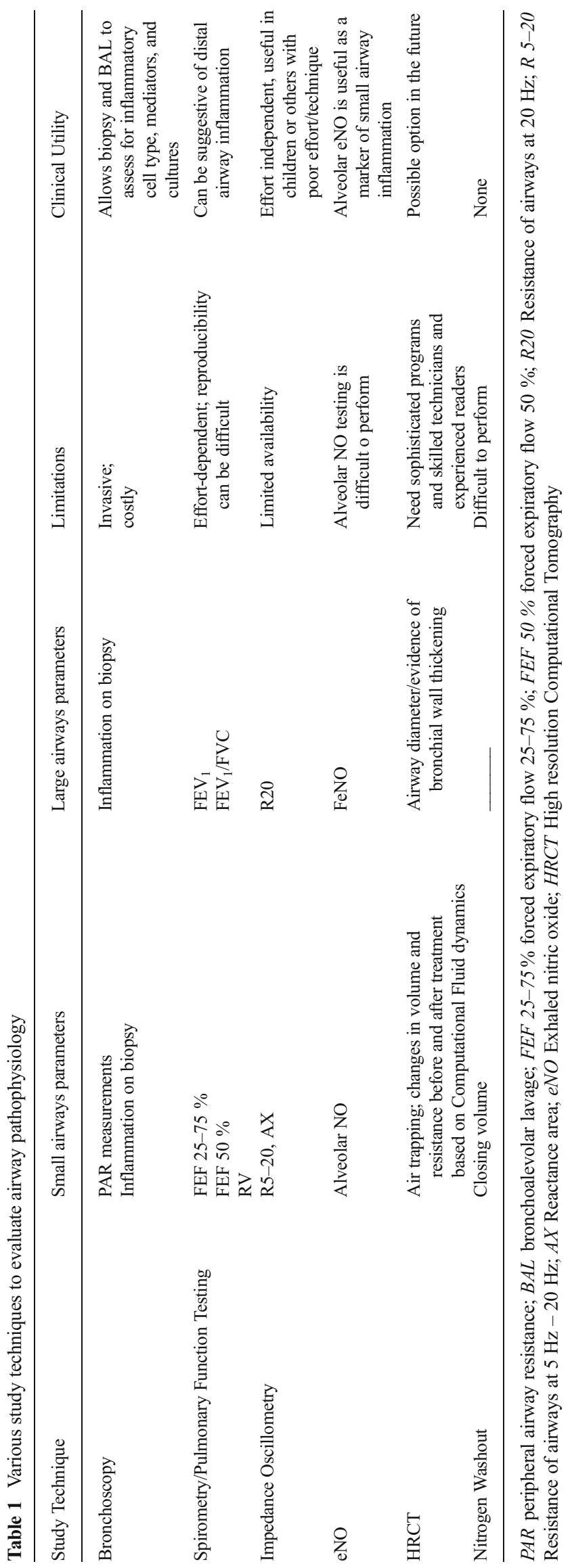




\section{Therapy and effect on inflammation}

Asthma is a clinical syndrome with airway inflammation resulting in expiratory airflow limitation with reversible airway obstruction and hyper-reactivity. Targeting the small airways with inhaled drug therapy is both patient and drug formulation-dependent. Respirable particle size is thought to be the biggest factor in drug delivery to distal airways, with the mass median aerodynamic diameter (MMAD) size being 1-2 microns, with 1.1-1.5 microns probably being ideal. Particles less than 1 micron are thought to be inhaled and exhaled without being deposited; as a result of Brownian motion, there is no settling. Particles greater than 2 microns have greater deposition in larger airways. This has been studied using isoproteronol in two different particle sizes and ipratropium of variety of sized particles [34, 35], with improved bronchodilation related to distal delivery with smaller particle size. Similarly, lung deposition of B2 agonists has been demonstrated to be greater at particle sizes of 1.5 microns versus 6 microns (56\% versus $46 \%$ ) [36].

In 1987, the Montreal Protocol mandated the elimination of the use of substances that depleted the ozone layer, and this included chlorofluorocarbons that had traditionally been used in metered dose inhalers (MDIs) as propellants. Alternate propellants were thus developed, with the principal one being hydrofluroalkanes (HFAs). Those propellants that were formulated to be in solution (rather than in suspension) resulted in a change in particle size and drug delivery. Breath actuated dry powder inhalers (DPIs) are the other main form of reformulation, but are of a larger particle size delivery [37]. Suspension MDIs have larger particle size than those in solution. Solution MDIs result in the drug being dissolved in the propellant or cosolvent. This results in smaller particle size. DPIs and suspension HFA MDIs exhibit particle sizes with MMAD greater than 2.6 micrometers. Particle size is inversely related to depth of penetration and the variety of options available is seen in Table 2 [38]. Several small studies assessing the Turbuhaler (a flow-driven multidose DPI that generates a 2 micron particle size at inhalation flow rate of $60 \mathrm{~L} /$ minute [39]) demonstrated that in comparison to MDI, there was improved lung deposition of budesonide (32\% versus $15 \%$ ) [40].

The change in propellant from chloroflurocarbon (CFC) to HFA resulted in beclomethasone being reformulated and having a mass median aerodynamic diameter of 1.1 micrometers versus 3.5-4.0 micrometers in the CFC version [41]. Leach and colleagues used technetium-99 m-radiolabeled beclomethasone dipropionate in CFC and HFA forms to demonstrate that smaller particle size is associated with improved delivery of the drug to peripheral airways. They noted the HFA-BDP delivered $55-60 \%$ to the airways and $29-30 \%$ in the oropharynx compared with CFC-BDP, which had 90-94\% deposition in the oropharynx and 4-7\% in the airways of healthy individuals [42]. There are other benefits to the new formulations, including
Table 2 Particle diameter size of various inhaled corticosteroids

\begin{tabular}{|c|c|}
\hline Drug & $\begin{array}{l}\text { Mean Median Aerodynamic } \\
\text { Diameter (microns) }\end{array}$ \\
\hline Fluticasone propionate - DPI & 6.5 \\
\hline Mometasone - DPI & 3.5 \\
\hline $\begin{array}{l}\text { Beclomethasone- CFC } \\
\text { (no longer available) }\end{array}$ & 3.5 \\
\hline Budesonide- DPI & 2.6 \\
\hline $\begin{array}{l}\text { Fluticasone propionate }-\mathrm{CFC} \text { and } \mathrm{HFA} \\
\text { (CFC formulation not available) }\end{array}$ & 2.6 \\
\hline $\begin{array}{l}\text { Flunisolide solution HFA } \\
\text { (not yet available) }\end{array}$ & 1.2 \\
\hline Beclomethasone - HFA & 1.1 \\
\hline Ciclesonide - HFA & 1.1 \\
\hline
\end{tabular}

DPI Dry powder inhaler; $C F C$ chloroflurocarbon; HFA hydrofluoroalkane

studies demonstrating that even with patient discoordination and in pediatric patients aged 5-17, there was greater delivery of ultra-fine particle size drug to the airways [38].

Several studies have since shown that there is equivalent clinical efficacy with reduced doses (one-half to one-third) of inhaled corticosteroids with the HFA preparations of both beclomethasone and flunisoilde [43, 44]. Despite fluticasone being traditionally considered a more potent corticosteroid, studies comparing the two in HFA aerosol formulations showed that the HFA formulation of beclomethasone resulted in equivalent asthma control as compared to similar doses of fluticasone [45, 46].

A more recent study looking at the use of extra-fine (EF) particle formulation of HFA-beclomethasone compared to CFC-beclomethasone has demonstrated that 1 year after initiating or stepping up therapy, patients receiving EF HFA-BDP were more likely to achieve asthma control, as compared to those on CFC-BDP. This suggested that particle size and deposition characteristics are relevant for effectiveness of asthma therapy. This study also demonstrated a lower dose needed for control with the EF-particle formulation [47••]. The measures used for evaluating treatment in all these studies have been $\mathrm{FEV}_{1}$, considered a measurement of large airway function. Yamaguchi and colleagues demonstrated improvement in small airway resistance impulse oscillometry measurements with the use of HFA-BDP as compared to CFC-BDP. They found that R5-R20 and AX progressively improved with HFABDP, achieving statistical significance at 12 weeks versus the CFC-BDP group, and the HFA-BPD was associated with a decline in methacholine hyper-reactivity [48].

As CFC inhalers are no longer available, it is more useful to look at differences in currently available treatment options. HFA-fluticasone propionate has a mass median aerodynamic diameter of 2.5 microns, as compared to HFA-ciclesonide and HFA-beclomethasone dipropopnate, which have a mass median aerodynamic diameter of 1.1 microns. A study looking at steroid naïve asthmatics in a primary care setting noted that patients 
given HFA-BDP had a statistically significant odds ratio of improved control, decreased short-acting beta-agonist (SABA) use, and less likelihood of needing additional therapy than with a larger particle size inhaled steroid, HFA-fluticasone [49••]. This study also demonstrated that if patients needed step-up therapy because of poor asthma control, the EF particle steroid had a trend of improvement similar to the initiation group, although not statistically significant. However, the EF particle size inhaled steroid group needed significantly less escalation of therapy $[49 \cdot \bullet]$.

A small study also looked at the effect of small particle ciclesonide compared to placebo and noted an improvement in alveolar nitric oxide and $\mathrm{CT}$ measurements of expiratory lung volumes after methacholine challenge [50]. Another small study compared beclomethasone/formoterol HFA MDI versus fluticasone/salmeterol DPI in ten patients with moderate persistent asthma and monitored small airways parameters of phase III of the nitrogen washout curve. They evaluated the closing volume as well as maximal expiratory flow rate at $50 \%$ of the FVC and demonstrated a significant improvement in these parameters when the patients were in the HFA MDI arm as compared to the DPI arm, at equipotent doses [51・•]. Vos and colleagues reported their findings evaluating the effect of beclomethasone/formeterol HFA using high resolution computerized tomography and computational fluid dynamics [52•]. They reported an improvement in small airway resistance using functional imaging in patients, even with well controlled asthma (by Asthma Control Test score and FEV 1 on spirometry) when they were started on or changed to treatment with EF particle sized beclomethasone/formoterol.

\section{Conclusion}

Asthma is a diagnosis of reversible airflow limitation that has significant heterogeneity in its presentation and response to treatment. While many factors are at play, targeting the small airways is likely to be beneficial in the majority of patients. This is now easier using the new formulations of metered dose inhalers that result in smaller particle size of corticosteroids with more effective distal delivery.

\section{Compliance with Ethics Guidelines}

Conflict of Interest Anita Trikha declares that she has no conflicts of interest.

Richard Martin is a paid consultant for Teva, AstraZeneca, MedImmune, Merck, Amgen, Sunovion, Novartis, and Genentech. He receives honoraria from Merck, Genengetch/Novartis, ACAAI, and Teva and receives royalties from UpToDate and Informa.

Human and Animal Rights and Informed Consent All studies by the authors involving human subjects were performed after approval by the appropriate Institutional Review Boards. Written informed consent was obtained from all participants.

\section{References}

Papers of particular interest, published recently, have been highlighted as:

- Of importance

- Of major importance

1. Hogg JC, Macklem PT, Thurlbeck WM. Site and nature of airway obstruction in chronic obstructive lung disease. New Engl J Med. 1968;278:1355-60.

2. Peters SP, Jones CA, Haselkorn T, et al. Real-world Evaluation of Asthma Control and Treatment (REACT): findings from a national Web-based survey. J Allergy Clin Immunol. 2007;119:1454-61.

3. Asthma in America: A landmark survey. Executive summary. SRBI. Available online at http://www.asthmainamerica.com/aaa_index. html; 1998.

4. Asthma Insight and Management (AIM) Survey. Available online at http://www.takingaimatasthma.com; 2009.

5. Bateman ED, Boushey HA, Bousquet J, et al. Can guideline-defined asthma control be achieved? The gaining optimal asthma control study. Am J Respir Crit Care Med. 2004;170:836-44.

6. Carroll N, Elliot J, Morton A, James A. The structure of large and small airways in nonfatal and fatal asthma. Am Rev Respir Dis. 1993;147: 405-10.

7. Hetzel MR, Clark TJ. Comparison of normal and asthmatic circadian rhythms in peak expiratory flow rate. Thorax. 1980;35:732-8.

8. Hetzel MR, Clark TJ, Branthwaite MA. Asthma: analysis of sudden deaths and ventilatory arrests in hospital. Br Med J. 1977;1:808-11.

9. Kraft M, Martin RJ, Wilson S, et al. Lymphocyte and eosinophil influx into alveolar tissue in nocturnal asthma. Am J Respir Crit Care Med. 1999;159:228-34.

10. Haley KJ, Sunday ME, Wiggs BR, et al. Inflammatory cell distribution within and along asthmatic airways. Am J Respir Crit Care Med. 1998;158:565-72.

11. Lambert RK, Wiggs BR, Kuwano K, et al. Functional significance of increased airway smooth muscle in asthma and COPD. J Appl Physiol. 1993;74:2771-81.

12. Schulman ES, Adkinson Jr NF, Newball HH. Cyclooxygenase metabolites in human lung anaphylaxis: airway vs. parenchyma. J Appl Physiol. 1982;53:589-95.

13. Hauber HP, Gotfried M, Newman K, et al. Effect of HFA-flunisolide on peripheral lung inflammation in asthma. J Allergy Clin Immunol. 2003;112:58-63.

14. Hyde DM, Hamid Q, Irvin CG. Anatomy, pathology, and physiology of the tracheobronchial tree: emphasis on the distal airways. J Allergy Clin Immunol. 2009;124:S72-7.

15. Irvin CG, Pak J, Martin RJ. Airway-parenchyma uncoupling in nocturnal asthma. Am J Respir Crit Care Med. 2000;161:50-6.

16. Kraft M, Pak J, Martin RJ, et al. Distal lung dysfunction at night in nocturnal asthma. Am J Respir Crit Care Med. 2001;163:1551-6.

17. Wagner EM, Liu MC, Weinmann GG, et al. Peripheral lung resistance in normal and asthmatic subjects. Am Rev Respir Dis. 1990;141:584-8.

18. Kraft M, Cairns CB, Ellison MC, et al. Improvements in distal lung function correlate with asthma symptoms after treatment with oral montelukast. Chest. 2006;130:1726-32.

19. Mahut B, Peiffer C, Bokov $P$, et al. Gas trapping is associated with severe exacerbation in asthmatic children. Respir Med. 2010;104:1230-3.

20. in't Veen JC, Beekman AJ, Bel EH, Sterk PJ. Recurrent exacerbations in severe asthma are associated with enhanced airway closure during stable episodes. Am J Respir Crit Care Med. 2000;161:1902-6.

21. McFadden Jr ER, Kiser R, DeGroot WJ. Acute bronchial asthma. Relations between clinical and physiologic manifestations. New Engl J Med. 1973;288:221-5. 
22. Zeidler MR, Goldin JG, Kleerup EC, et al. Small airways response to naturalistic cat allergen exposure in subjects with asthma. J Allergy Clin Immunol. 2006;118:1075-81.

23. Kaminsky DA, Irvin CG, Gurka DA, et al. Peripheral airways responsiveness to cool, dry air in normal and asthmatic individuals. Am J Respir Crit Care Med. 1995;152:1784-90.

24. Sutherland ER, Martin RJ, Bowler RP, et al. Physiologic correlates of distal lung inflammation in asthma. J Allergy Clin Immunol. 2004;113:1046-50.

25. - Shi Y, Aledia AS, Tatavoosian AV, et al. Relating small airways to asthma control by using impulse oscillometry in children. J Allergy Clin Immunol. 2012;129:671-8. Good demonstration of a new technique to assess small airways in a population traditionally difficult to assess with traditional office spirometry.

26. Bhansali PV, Irvin CG, Dempsey JA, et al. Human pulmonary resistance: effect of frequency and gas physical properties. J Appl Physiol. 1979;47:161-8.

27. Lee JH, Lee YW, Shin YS, et al. Exercise-induced airway obstruction in young asthmatics measured by impulse oscillometry. J Investig Allergol Clin Immunol. 2010;20:575-81.

28. - Liang BM, Lam DC, Feng YL. Clinical applications of lung function tests: a revisit. Respirology. 2012;17:611-9. Good review of available techniques available to assess the airways with description of limitations.

29. • van der Wiel E, ten Hacken NH, Postma DS, van den Berge M. Small-airways dysfunction associates with respiratory symptoms and clinical features of asthma: a systematic review. J Allergy Clin Immunol. 2013;131:646-57. Excellent review of the literature relating to small airways and limitations in evaluating the distal lung.

30. Scichilone N, Battaglia S, Taormina S, et al. Alveolar nitric oxide and asthma control in mild untreated asthma. J Allergy Clin Immunol. 2013;131:1513-7.

31. Berry M, Hargadon B, Morgan A, et al. Alveolar nitric oxide in adults with asthma: evidence of distal lung inflammation in refractory asthma. Eur Respir J. 2005;25:986-91.

32. Okazawa M, Muller N, McNamara AE, et al. Human airway narrowing measured using high resolution computed tomography. Am J Respir Crit Care Med. 1996;154:1557-62.

33. Verbanck S, Schuermans D, Paiva M, Vincken W. Nonreversible conductive airway ventilation heterogeneity in mild asthma. J Appl Physiol. 2003;94:1380-6.

34. Patel P, Mukai D, Wilson AF. Dose-response effects of two sizes of monodisperse isoproterenol in mild asthma. Am Rev Respir Dis. 1990;141:357-60.

35. Zanen P, Go LT, Lammers JW. Optimal particle size for beta 2 agonist and anticholinergic aerosols in patients with severe airflow obstruction. Thorax. 1996;51:977-80.

36. Usmani OS, Biddiscombe MF, Barnes PJ. Regional lung deposition and bronchodilator response as a function of beta2-agonist particle size. Am J Respir Crit Care Med. 2005;172:1497-504.

37. Sutherland ER, Martin RJ. Targeting the distal lung in asthma: do inhaled corticosteroids treat all areas of inflammation? Treat Respir Med. 2005;4:223-9.

38. Leach C, Colice GL, Luskin A. Particle size of inhaled corticosteroids: does it matter? J Allergy Clin Immunol. 2009;124:S88-93.
39. Tamura G, Sakae H, Fujino S. In vitro evaluation of dry powder inhaler devices of corticosteroid preparations. Allergol Int. 2012;61: $149-54$.

40. Thorsson L, Edsbacker S, Conradson TB. Lung deposition of budesonide from Turbuhaler is twice that from a pressurized metered-dose inhaler P-MDI. Eur Respir J. 1994;7:1839-44.

41. Vanden Burgt JA, Busse WW, Martin RJ, et al. Efficacy and safety overview of a new inhaled corticosteroid, QVAR (hydrofluoroalkanebeclomethasone extrafine inhalation aerosol), in asthma. J Allergy Clin Immunol. 2000;106:1209-26.

42. Leach CL, Davidson PJ, Boudreau RJ. Improved airway targeting with the CFC-free HFA-beclomethasone metered-dose inhaler compared with CFC-beclomethasone. Eur Respir J. 1998;12:1346-53.

43. Corren J, Nelson H, Greos LS, et al. Effective control of asthma with hydrofluoroalkane flunisolide delivered as an extrafine aerosol in asthma patients. Ann Allergy Asthma Immunol. 2001;87:405-11.

44. Gross G, Thompson PJ, Chervinsky P, Vanden BJ. Hydrofluoroalkane134a beclomethasone dipropionate, 400 microg, is as effective as chlorofluorocarbon beclomethasone dipropionate, 800 microg, for the treatment of moderate asthma. Chest. 1999;115:343-51.

45. Aubier M, Wettenger R, Gans SJ. Efficacy of HFA-beclomethasone dipropionate extra-fine aerosol (800 microg day $(-1))$ versus HFAfluticasone propionate $(1000$ microg day $(-1))$ in patients with asthma. Respir Med. 2001;95:212-20.

46. Fairfax AJ. The relative clinical effectiveness of HFA-BDP and fluticasone propionate in asthma. Respir Med. 2000;94(Suppl D): S31-6.

47. •• Barnes N, Price D, Colice G, et al. Asthma control with extrafineparticle hydrofluoroalkane-beclometasone vs. large-particle chlorofluorocarbon-beclometasone: a real-world observational study. Clin Exp Allergy. 2011;41:1521-32. Initial evidence of effects on asthma control and treatment as it relates to smaller drug particle size.

48. Yamaguchi M, Niimi A, Ueda T, et al. Effect of inhaled corticosteroids on small airways in asthma: investigation using impulse oscillometry. Pulm Pharmacol Therapeut. 2009;22:326-32.

49. •- Price D, Martin RJ, Barnes N, et al. Prescribing practices and asthma control with hydrofluoroalkane-beclomethasone and fluticasone: a real-world observational study. J Allergy Clin Immunol. 2010;126:511-8. e1-10. Evidence for the benefits of targeting the distal lung to acheive improvement in control.

50. Cohen J, Douma WR, ten Hacken NH, et al. Ciclesonide improves measures of small airway involvement in asthma. Eur Respir J. 2008;31:1213-20.

51. • Corda L, Gardenghi GG, Modina D, et al. Effects on small airway obstruction of long-term treatments with beclomethasone/formoterol hydrofluoroalkane (metered-dose inhaler) versus fluticasone/ salmeterol (dry-powder inhaler) in asthma: a preliminary study. Allergy Asthma Proc. 2011;32:29-34. Again, evidence supporting the utility of targeting the smaller airways.

52. - Vos W, De Backer J, Poli G, et al. Novel functional imaging of changes in small airways of patients treated with extrafine beclomethasone/formoterol. Respiration. 2013. Evidence for utility of emerging technological advances that could be clinically useful in years to come. 